\title{
Remodeling of epithelial cells and basement membranes in a corneal deficiency model with long-term follow-up
}

\author{
Sumako Kameishi ${ }^{1,2}$, Hiroaki Sugiyama ${ }^{2}$, Masayuki Yamato ${ }^{2}$, Yoshikazu Sado ${ }^{3}$, Hideo Namiki ${ }^{1}$, Takashi Kato ${ }^{1}$ and \\ Teruo Okano²
}

The ocular surface consists of the cornea, conjunctiva, and the limbus that is located in the transitional zone between the cornea and conjunctiva. The corneal epithelial cells are generated through the mitosis of corneal epithelial stem cells in the limbus. This study investigated a rabbit corneal deficiency model prepared by the surgical removal of the corneal and limbal epithelia, which express cytokeratin 12 (K12). After the surgery, K13-expressing conjunctival epithelium migrated onto the corneal surface and completely covered the surface, leading to neovascularization and corneal opacification. However, at 24 and 48 weeks after the surgery, K12-expressing cornea-like cells reappeared on the model ocular surface. These cells formed an island surrounded by invaded conjunctiva and were isolated from the limbus. Interestingly, in the 24-week model surface, $\alpha 1$ (IV) and $\alpha 2$ (IV) collagen chains, which are normally found in the basement membrane of the native limbus and conjunctiva, and not in the cornea, were continuously deposited throughout the entire basement membrane, including the basement membrane under cornea-like cells. By contrast, in the 48-week model surface, $\alpha 1$ (IV) and $\alpha 2$ (IV) collagen chains were absent from the basement membrane beneath the central part of cornea-like cells and were localized below the invaded conjunctiva and the transitional zone between cornea-like cells and the invaded conjunctiva, which had similar distribution to the native ocular basement membrane. Moreover, K12, K14, p63, vimentin, and $\alpha 1$ (IV) and $\alpha 2$ (IV) collagen chains, which are colocalized in the native limbus, were all present at the transitional zone of the 48-week model surface. Therefore, a limbus-like structure appeared to be reconstructed on the surface of the 48-week model as a stem cell niche. This study should aid in the understanding of human corneal deficiency, the correlation between the epithelial cell phenotype and the composition of the basement membrane, and the epithelial stem cell niche.

Laboratory Investigation (2015) 95, 168-179; doi:10.1038/labinvest.2014.146; published online 22 December 2014

The surface of the cornea is covered by a stratified squamous epithelium consisting of three to five nonkeratinized epithelial cell layers. ${ }^{1-3}$ Corneal epithelial cells are formed through mitosis at the basal layer and then move up to the surface region and slough off from the superficial layer. This turnover process is known to occur over 2 weeks. Homeostasis of the corneal surface is maintained by the generation of new cells from the stem cells of the limbus, which is located at the transitional zone between the cornea and the conjunctiva. ${ }^{4-8}$ When corneal epithelial stem cells have been irreversibly injured by severe trauma or diseases such as in Stevens-Johnson syndrome or ocular pemphigoid, the adjacent conjunctival tissue covers the ocular surface, leading to corneal vascularization, opacification, and severe visual loss. ${ }^{9-11}$ These pathological characteristics are considered to represent corneal epithelial stem cell deficiencies. The rabbit corneal deficiency model has been reported to be produced by the surgical removal of the corneal and limbal epithelia and the topical application of 1-n-heptanol onto the cornea and the limbus. ${ }^{12}$ In the reported corneal deficiency model, opacification of the corneal surface, conjunctival invasion with goblet cells, and neovascularization have been

\footnotetext{
${ }^{1}$ Graduate School of Advanced Science and Engineering, Waseda University, Tokyo, Japan; ${ }^{2}$ Institute of Advanced Biomedical Engineering and Science, Tokyo Women's Medical University, Tokyo, Japan and ${ }^{3}$ Division of Immunology, Shigei Medical Research Institute, Okayama, Japan

Correspondence: Dr/Professor M Yamato, Institute of Advanced Biomedical Engineering and Science, Tokyo Women's Medical University, TWIns 8-1 Kawada-cho, Shinjuku-ku, Tokyo 162-8666, Japan or Dr/Professor T Kato, Graduate School of Advanced Science and Engineering, Waseda University, 2-2 Wakamatsu, Shinjuku, Tokyo 162-8480, Japan.

E-mail: yamato.masayuki@twmu.ac.jp or tkato@waseda.jp

Received 29 January 2014; revised 20 October 2014; accepted 22 October 2014
} 
observed to be comparable to the processes in human corneal stem cell deficiency. ${ }^{12-14}$ In this study, rabbit corneal deficiency models were prepared to investigate the remodeling of corneal epithelia and their basement membranes.

In the wound-healing process, corneal epithelial stem cells provide differentiated cells for reconstructing the epithelium. Corneal epithelial stem cells are regulated by their own microenvironment, including cell-cell and cell-extracellular matrix (ECM), known as the 'stem-cell niche. ${ }^{15-17}$ Previous study demonstrated that some growth factors and morphogenetic factors bind directly to particular ECMs and regulate cell proliferation and differentiation. ${ }^{18}$ Moreover, the variations in basement membranes are known to depend on the tissue. On the ocular surface, the cornea, which contains terminally differentiated cells, and the limbus, which contains stem/progenitor cells, reside on the basement membranes with different components. ${ }^{19}$ Therefore, the basement membrane of the limbus is presumed to regulate the proliferation and differentiation of corneal epithelial stem cells.

The aim of this study was to investigate the remodeling of the corneal epithelium and basement membrane in a corneal deficiency model. Specifically, the ocular surfaces were examined, by immunohistochemistry with $\alpha$-chain-specific anti-type-IV collagen antibodies and putative stem/progenitor cell markers, to determine the correlation between the epithelial cell phenotype and the composition of the basement membrane.

\section{MATERIALS AND METHODS}

\section{Preparation of the Rabbit Corneal Deficiency Model}

New Zealand white rabbits weighing $\sim 2.5 \mathrm{~kg}$ each were treated in accordance with the Association for Research in Vision and Ophthalmology (ARVO) Statement for the Use of Animals in Ophthalmic and Vision Research and with the experimental procedures approved by the Committee for the Tokyo Women's Medical University. After anesthesia, the entire corneal epithelium, including the limbus and conjunctival tissue $3 \mathrm{~mm}$ beyond the transitional zone between the cornea and conjunctiva, was completely and surgically removed, exposing the stroma. After mechanical debridement, the ocular surface was treated with the topical application of $1-n$-heptanol for $5 \mathrm{~min}$. Antibiotic ( $0.3 \%$ ofloxacin) and steroid $(0.1 \%$ betamethasone $)$ eye drops were applied once a day for 1 week. ${ }^{12,20}$ At 4, 24, and 48 weeks after the surgery, the rabbits were killed using an overdose of anesthetic agent (pentobarbital), and the eyes were enucleated for histochemical analysis.

\section{Immunohistochemistry}

Normal and rabbit corneal deficiency model tissues at 4, 24, and 48 weeks after the surgery were collected, embedded in optimum-cutting-temperature compound, and processed into $5-\mu \mathrm{m}$ frozen sections. After drying for $1 \mathrm{~h}$ at room temperature, the tissue sections were washed with Dulbecco's phosphate-buffered saline (PBS; Sigma-Aldrich, St Louis, $\mathrm{MO}$, USA), incubated with $3 \% \mathrm{H}_{2} \mathrm{O}_{2}$ solution to inactivate internal peroxidase, and treated with PBS containing 5\% donkey serum (Sigma-Aldrich) and 0.3\% Triton X (SigmaAldrich) for $1 \mathrm{~h}$ to block nonspecific reactions. For the immunostainings of type IV collagen $\alpha$-chain-specific rat monoclonal antibodies, sections were treated with a mixture of $0.1 \mathrm{~mol} / \mathrm{l} \mathrm{KCl}$ and $0.1 \mathrm{~mol} / \mathrm{l} \mathrm{HCl}$ solution ( $\mathrm{pH} 3.5$ ) for $10 \mathrm{~min}$ at room temperature to denature epitopes. ${ }^{21}$ Primary antibodies were incubated overnight at $4{ }^{\circ} \mathrm{C}$. Details of the procedures using various antibodies are shown in Table 1. The secondary antibodies were horseradish peroxidaseconjugated anti-mouse, anti-goat, and anti-rat IgG (1:1000 dilution; Jackson Immuno Research Laboratories, West Grove, PA, USA), Alexa 568-conjugated anti-mouse IgG (1:500 dilution; Molecular Probes, Eugene, OR, USA), and Alexa 488-conjugated streptavidin (Molecular Probes). The sections were examined with an upright microscope and confocal microscopes (Carl Zeiss Meditec, Jena, Germany).

\section{RESULTS \\ Condition of the Ocular Surfaces and Epithelial Cell Phenotype}

The rabbit corneal deficiency model was prepared by $1-n-$ heptanol treatment after the surgical removal of the corneal and limbal epithelial layers, including the basement membrane. The epithelial defect was diagnosed with fluorescein staining because native corneal epithelium is completely protected from fluorescein penetration by its tight junctions. ${ }^{22}$ At 1 week post surgery, the corneal stroma was stained well with fluorescein (Figure 1). After 4 weeks, neovascularization developed into the corneal region from the conjunctiva, and fibrous tissues invaded. Fluorescein staining was observed in the central part of the corneal deficiency model surface except at the peripheral cornea, indicating that epithelial cells migrated into the corneal surface from the conjunctiva (Figure 1). At $\sim 6$ weeks post surgery, epithelial tissue covered the entire surface of the cornea with angiogenesis, and prevented fluorescein staining even after 24 and 48 weeks (Figure 1). Corneal deficiency model rabbits were killed at 4, 24, and 48 weeks, and the ocular surfaces were examined histologically. Native corneal stroma is avascular and consists of uniform and linear lamellae of fibrillar collagen. ${ }^{23,24}$ By contrast, the stroma in the corneal deficiency model was vascularized (Figures $2 \mathrm{~b}$ and $\mathrm{c}$, asterisks), and the collagen fibrils were randomly oriented (Figure $2 \mathrm{a}-\mathrm{c}$ ), as has also been observed in the pannus of human SJS patients. ${ }^{25}$ The 4-week corneal deficiency model surface showed expression of cytokeratin 13 (K13; Figure 2d), a conjunctival epithelial marker, ${ }^{26,27}$, and mucin 5AC (Muc5; Figure 2g), a goblet cell marker. Moreover, K12, a corneal epithelial cell marker, was absent from the 4-week corneal deficiency model surface (Figure 2j). These data suggested that conjunctival epithelial cells were migrating into the corneal region after the removal of the limbal and corneal epithelia. The presence of goblet cells has been considered evidence for the migration of conjunctival epithelium onto corneal stroma, ${ }^{28}$ termed 
Table 1 Primary antibody list

\begin{tabular}{|c|c|c|c|c|}
\hline Primary antibody & Clonality & Distribution & Dilution & Source \\
\hline Cytokeratin 12 (K12) & Goat polyclonal & Corneal epithelium & $1: 100$ & Santa Cruz Biotechnology, Santa Cruz, CA, USA \\
\hline Cytokeratin 13 (K13) & Mouse monoclonal & $\begin{array}{l}\text { Mucosal epithelium } \\
\text { (including conjunctival epithelium) }\end{array}$ & $1: 100$ & Progen Biotechnik, Heidelberg, Germany \\
\hline Mucin 5AC (Muc5) & Mouse monoclonal & Conjunctival goblet cell & $1: 100$ & Invitrogen, Carlsbad, CA, USA \\
\hline Cytokeratin 14 (K14) & Mouse monoclonal & Basal epithelial cell & $1: 100$ & Abcam, Cambridge, UK \\
\hline p63 & Mouse monoclonal & Epithelial stem/progenitor cell & $1: 100$ & Santa Cruz Biotechnology \\
\hline Vimentin & Mouse monoclonal & $\begin{array}{l}\text { Mesenchymal cell and epithelial } \\
\text { stem/progenitor cell }\end{array}$ & $1: 100$ & Dako, Carpinteria, USA \\
\hline Type IV collagen & Mouse monoclonal & Basement membrane and vessel & $1: 100$ & Daiichi Fine Chemical, Toyama, Japan \\
\hline$\alpha 1(\mathrm{IV})$ collagen chain & Rat monoclonal & Basement membrane and vessel & $1: 20$ & Gifted from Yoshikazu Sado \\
\hline$\alpha 2(\mathrm{IV})$ collagen chain & Rat monoclonal & Basement membrane and vessel & $1: 5$ & Gifted from Yoshikazu Sado \\
\hline$\alpha 3(\mathrm{IV})$ collagen chain & Rat monoclonal & Basement membrane & $1: 5$ & Gifted from Yoshikazu Sado \\
\hline$\alpha 4(\mathrm{IV})$ collagen chain & Rat monoclonal & Basement membrane & $1: 5$ & Gifted from Yoshikazu Sado \\
\hline$\alpha 5$ (IV) collagen chain & Rat monoclonal & Basement membrane & $1: 5$ & Gifted from Yoshikazu Sado \\
\hline
\end{tabular}
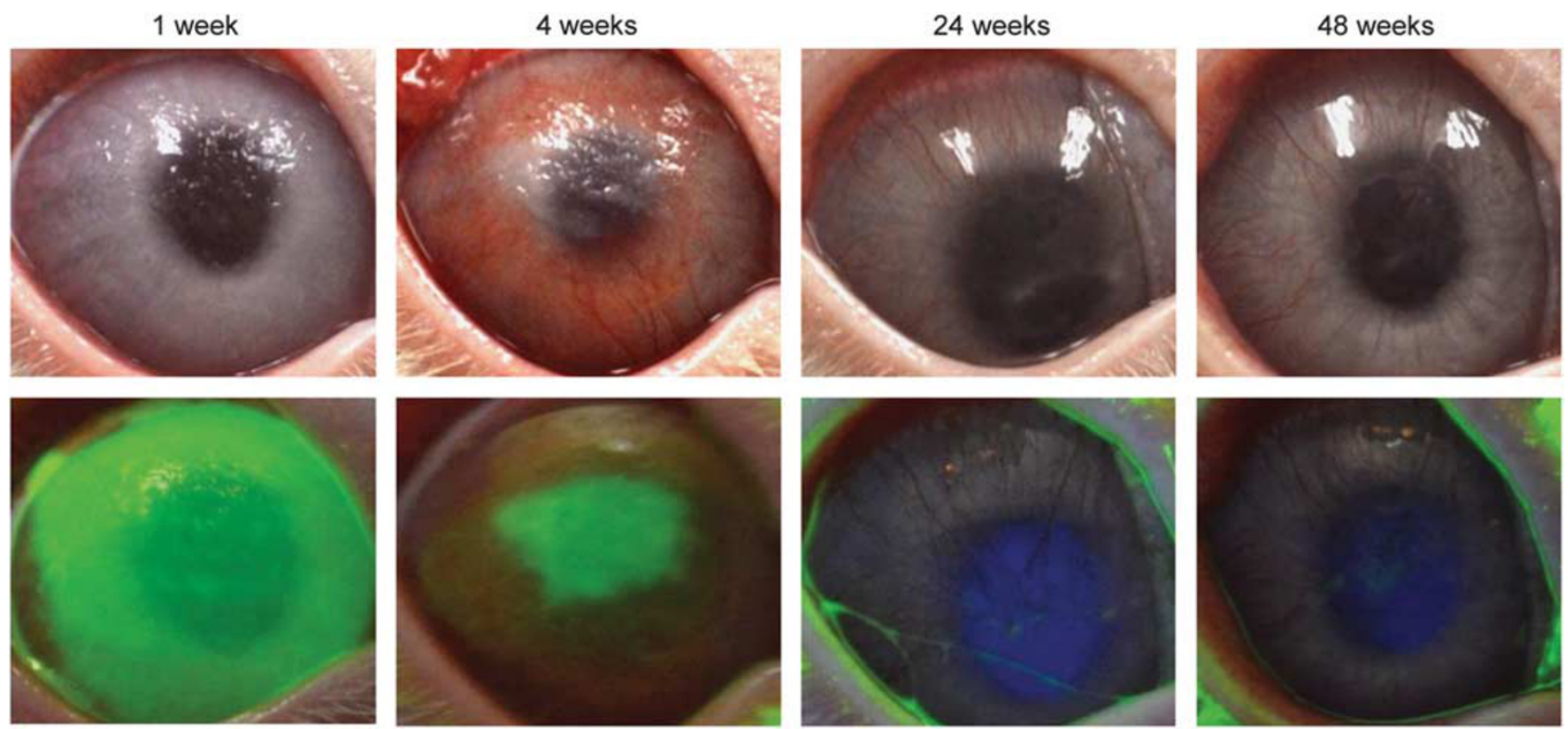

Figure 1 Time course observations in a rabbit corneal deficiency model with long-term follow-up. The upper and lower rows show photographs of the time course of the ocular surfaces of the rabbit corneal deficiency model before and after fluorescein staining, respectively.

invaded conjunctiva, and this occurrence has been theorized to be similar to some ocular surface diseases including corneal stem cell deficiency and pterygium. ${ }^{29,30}$ The rabbit corneal deficiency model in this study is believed to reflect the clinical status of corneal stem cell deficiency based on three lines of evidence: vascularized stroma, the expression of K13, and the presence of goblet cells.

After 24 weeks of follow-up, the central region of the epithelium was observed to be thicker (Figure 2b, black line). To investigate the epithelial cell phenotype, immuno- histochemical analyses with K13, Muc5, and K12 were performed. In the 24-week model surface, K13 expression was observed in the peripheral region of the corneal surface (Figure 2e, left side). However, K13 expression was not observed in the thicker epithelium at the central part of the cornea (Figure 2e, black line), which had a curved length of $\sim 2.5 \mathrm{~mm}$ (Supplementary Figure 1). Moreover, Muc5expressing conjunctival goblet cells were not detected in the central thicker epithelium, whereas the peripheral region contained Muc5-expressing conjunctival goblet cells (Figure 2h). 


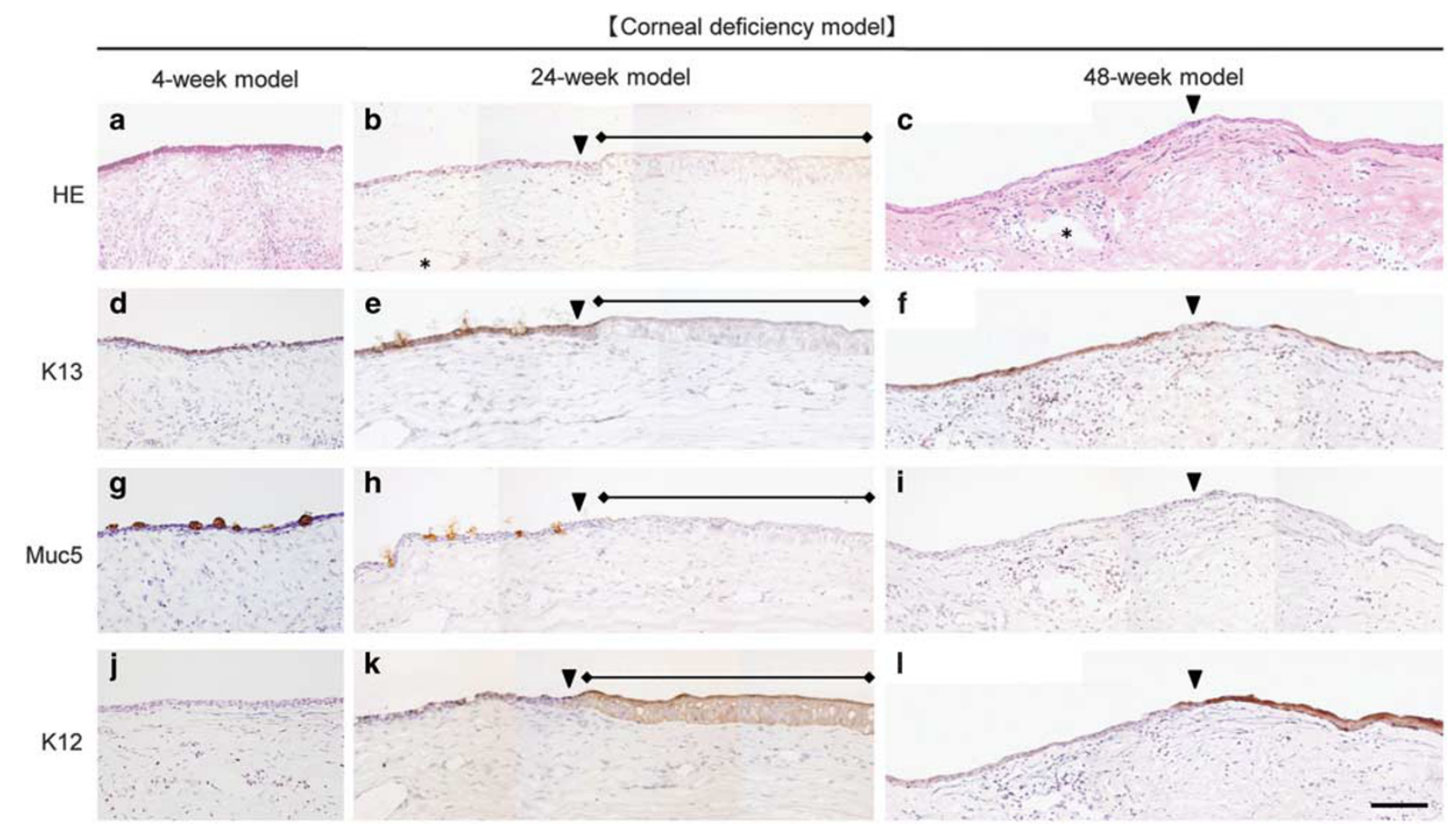

Figure 2 Localized changes in keratins and mucin in the epithelial layer after the removal of the corneal and limbal epithelia. Hematoxylin-eosin staining (a-c) and immunohistochemistry with antibodies for cytokeratin 13 (K13), a conjunctival epithelial marker (d-f), mucin 5AC (Muc5), a goblet cell marker (g-i), and K12, a corneal epithelial cell marker (j-I), on 4-, 24-, and 48-week model surfaces. Scale bar $=25 \mu \mathrm{m}$.

Remarkably, despite the removal of the K12-expressing corneal epithelium to prepare the model, K12-positive/K13negative cells were found (Figure 2k), which had hardly been detected on the 4-week model surface (Figure 2j). The K12expressing cornea-like cell layer was thicker (Figure 2, black bar) than K12-negative cell region of 24-week model surface, and was observed to be surrounded by invaded conjunctiva as well as being isolated from the limbus (Supplementary Figure 1). On the 48-week model surface, expression of K13 was observed in invaded conjunctiva (Figure $2 \mathrm{f}$, left side). Although K13 expression was discontinuously observed in the only superficial layer at the central part of the cornea (Figure 2f, right side) and Muc5-expressing cell was not contained (Figure 2i, right side), K12 expression was observed in the all epithelial cell layers (Figure 2l, right side). This result is consistent with the observations in the 24-week model surface. The curved length of K12-expressing cells of 48-week model surface was $\sim 7.5 \mathrm{~mm}$ (Supplementary Figure 2).

\section{Correlation between the Epithelial Cell Phenotype and the Composition of Type IV Collagen}

Collagen is a protein superfamily that has a characteristic triple helix domain composed of three $\alpha$-chains derived from the corresponding genes. In the case of type IV collagen, six genetically distinct $\alpha$-chain genes have been identified. ${ }^{21,31} \mathrm{~A}$ subtype of type IV collagen, $\alpha 1-\alpha 1-\alpha 2$ (IV), is common as a major component of the basement membrane. $\alpha 1-\alpha 1-\alpha 2$ (IV) collagen has been previously reported to exist in the basement membrane of the native human ocular surface under the conjunctival and limbal epithelia but to be absent from the central cornea. ${ }^{19,32} \alpha 3-\alpha 4-\alpha 5$ (IV) collagen is specifically formed in the basement membrane under the corneal epithelium. ${ }^{19,32}$ In addition, $\alpha 5-\alpha 5-\alpha 6$ (IV) is formed in the basement membrane under the entire ocular surface, from the conjunctiva to the central cornea. ${ }^{19,32}$ In this study, native rabbit ocular surfaces were stained with monoclonal antibodies recognizing $\alpha 1$ (IV), $\alpha 2(\mathrm{IV}), \alpha 3(\mathrm{IV})$, $\alpha 4(\mathrm{IV})$, and $\alpha 5(\mathrm{IV})$ collagen chains. These antibodies are raised against amino-acid residues in the carboxy-terminal domain of the chain and have been previously confirmed to exhibit cross-reactivity for rabbit counterparts. ${ }^{21}$ In the native rabbit ocular surface, $\alpha 1$ (IV) (Figures $3 a-c$ ) and $\alpha 2(I V)$ (Figures $3 \mathrm{~d}-\mathrm{f}$ ) collagen chains were observed in the conjunctiva and the limbus and were absent from the cornea. Staining for the $\alpha 3(\mathrm{IV})$ collagen chain was not observed in the basement membrane of the entire ocular surface, detected only in the some microvessels (Figures 3g-i). $\alpha 4$ (IV) collagen chain was weakly detected only in the limbal basement membrane (Figures 3j-l). $\alpha 5$ (IV) collagen chain was located throughout the ocular surface, from the conjunctiva to the central cornea (Figures $3 \mathrm{~m}-\mathrm{o}$ ). The dis- 


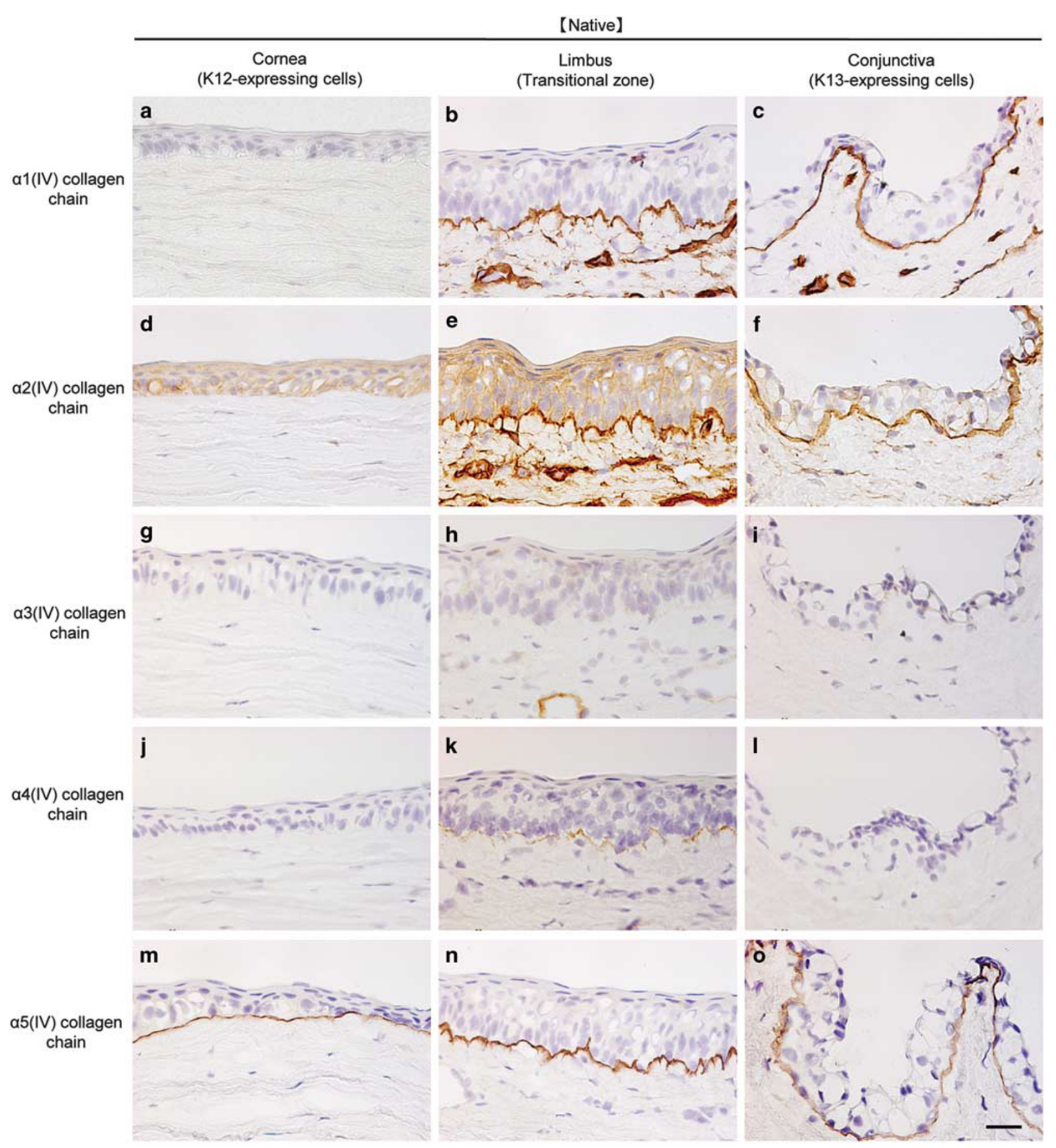

Figure 3 Distribution of type IV collagen $\alpha$-chain isoforms on the native rabbit ocular surface. Immunohistochemical staining of native rabbit cornea, limbus, and conjunctiva with $\alpha 1(\mathrm{IV})(\mathbf{a}-\mathbf{c}), \alpha 2$ (IV) (d-f), $\alpha 3(\mathrm{IV})(\mathbf{g}-\mathbf{i}), \alpha 4(\mathrm{IV})(\mathbf{j}-\mathrm{I})$, and $\alpha 5(\mathrm{IV})(\mathbf{m}-\mathbf{o})$ collagen chains. Scale bar $=25 \mu \mathrm{m}$.

tribution of type IV collagen $\alpha$-chains in native rabbit ocular surfaces is summarized in Table 2.

In the 24- and 48-week corneal deficiency models, immunohistochemical staining with anti- $\alpha 5$ (IV) collagen chain antibody was performed on serial sections stained with antibodies against K12 and K13. $\alpha 5$ (IV) collagen chain was abundantly deposited in the basement membrane beneath K12-expressing cornea-like cells (Figures 4a and d: arrow head) with faint or negative deposition in the basement membrane underlying invaded conjunctiva (Figures $4 \mathrm{c}$ and $\mathrm{f}$ ). Therefore, these findings demonstrated that the composition of the basement membrane beneath cornea-like cells was different from that of the surrounding area. 
In addition, to investigate the correlation between the epithelial cell phenotype and the composition of the basement membrane clearly, double staining for $\alpha 1$ (IV) and $\alpha 2$ (IV) collagen chains and K12 was performed. In the native ocular surface, $\alpha 1$ (IV) and $\alpha 2$ (IV) collagen chains were deposited in the basement membrane under the limbal and conjunctival epithelia and were absent from the basement membrane under the central corneal epithelium (Figures $5 \mathrm{a}-\mathrm{c}$ ). On the 24-week model surface, $\alpha 1$ (IV) and $\alpha 2$ (IV) collagen chains were continuously deposited throughout the whole ocular surface, including under cornea-like cells (Figures $5 \mathrm{~d}-\mathrm{f}$ ). On the 48-week model surface, $\alpha 1$ (IV) and $\alpha 2$ (IV) collagen chains were deposited in the basement membrane under the transitional zone and invaded conjunctiva (Figures $5 \mathrm{~h}$ and i) and were absent from the basement membrane under the central region of the cornea-like cells (Figure 5g). Moreover, $\alpha 1$ (IV) and $\alpha 2$ (IV) collagen chains were also expressed on the vessels in the stroma, including under the cornea-like cells (Figure 5, asterisks).

Table 2 Distribution of type IV collagen $\alpha$-chains on ocular surface

\begin{tabular}{lcccc}
\hline & Cornea & Limbus & Conjunctiva & Microvessels \\
\hline$\alpha 1(\mathrm{IV})$ collagen chain & - & ++ & ++ & ++ \\
$\alpha 2(\mathrm{IV})$ collagen chain & - & ++ & ++ & ++ \\
$\alpha 3(\mathrm{IV})$ collagen chain & - & - & - & $+/-$ \\
$\alpha 4(\mathrm{IV})$ collagen chain & - & + & - & - \\
$\alpha 5(\mathrm{IV})$ collagen chain & ++ & ++ & ++ & $+/-$ \\
\hline
\end{tabular}

\section{Reconstruction of a Limbus-like Structure on the 48-week Model Surface}

On the native ocular surface, the expression of K14, a basal epithelial cell marker, and p63, a putative stem/progenitor cell marker, are known to colocalize in the limbal basal epithelial cell layer. By contrast, K12, a marker of differentiated corneal epithelial cells, is expressed in the full thickness of the central cornea and in the suprabasal to superficial layers of the limbus without a basal cell layer. ${ }^{33}$ First, histological double staining was performed with antibodies against K14 and $\alpha 1$ (IV) and $\alpha 2$ (IV) collagen chains. In the 48-week model surface of the transitional zone between cornea-like cells and invaded conjunctiva, K14expressing cells were found to localize on the basement membrane containing $\alpha 1$ (IV) and $\alpha 2$ (IV) collagen chains (Figure 6d); K14-expressing cells and $\alpha 1$ (IV) and $\alpha 2$ (IV) collagen chains were also observed in the native limbus (Figure 6a). However, the expressions of K14 and $\alpha 1$ (IV) and $\alpha 2$ (IV) collagen were not observed in the central part of the cornea-like cells of the model surface, except for in the blood vessels (Supplementary figure 3d). Next, the expression patterns of K12, K14, and p63 were examined. In the native limbus, although the basal epithelium expressed K14 and p63, the upper cell layers expressed K12 (Figures $6 \mathrm{~b}$ and c). At the transitional zone of the 48 -week model surface, the basal cell layer expressed K14 and p63, and the upper cell layers expressed $\mathrm{K} 12$, which were similar to the native limbus (Figures 6e and f). By contrast, in the native cornea and in the central region of cornea-like cells of the 48-week model surface, the expressions of K14 and p63 were not observed (Supplementary Figure 3b, c, e, and f).

\section{【Corneal deficiency model】}

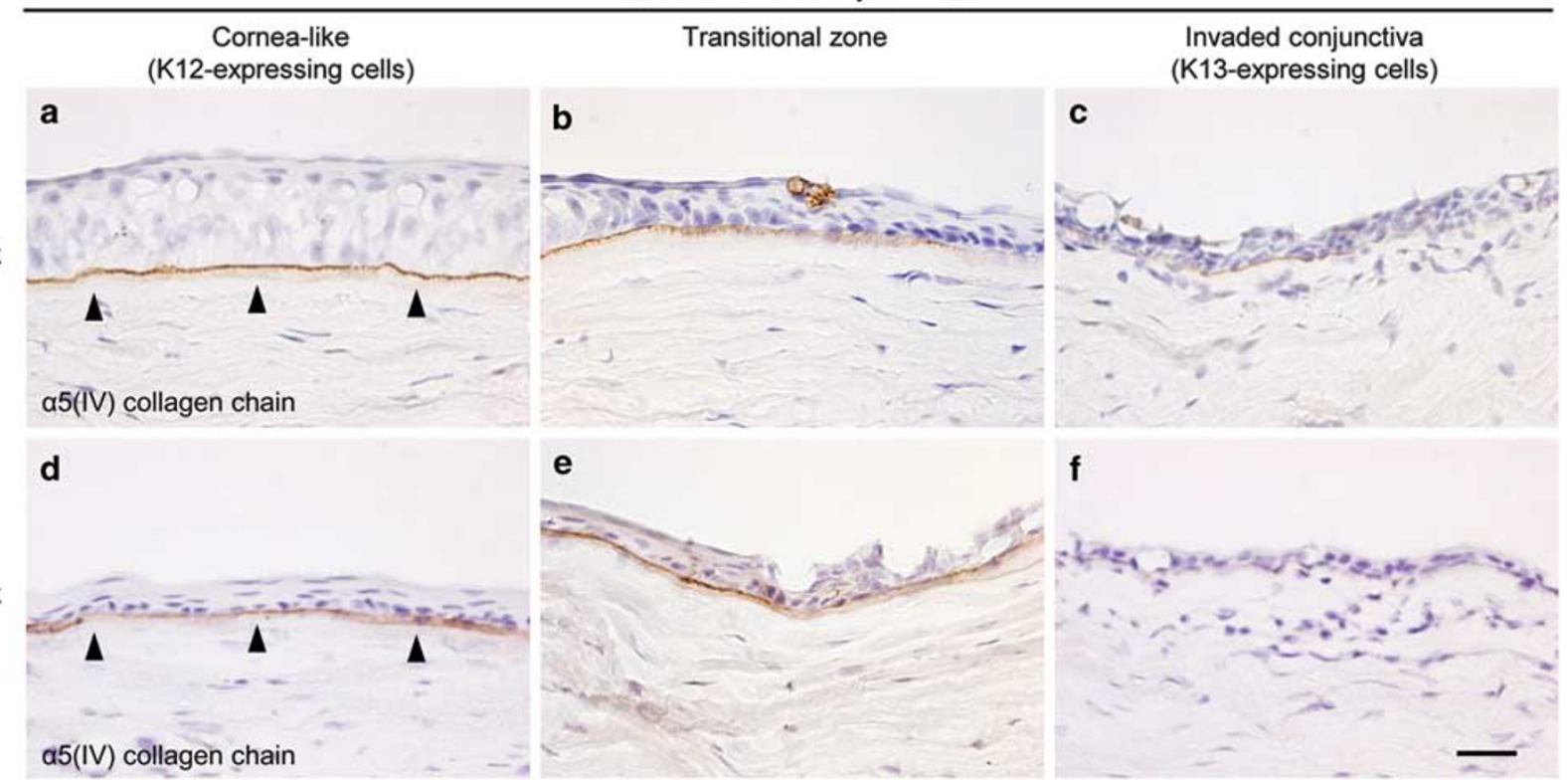

Figure 4 Localization of $\alpha 5$ (IV) collagen chain under cornea-like cells and invaded conjunctiva in the rabbit corneal deficiency model. Immunohistochemical staining for $\alpha 5$ (IV) collagen chain on 24-week (a-c) and 48-week model (d-f) surfaces. The examined regions of K12-expressing cornea-like cells $(\mathbf{a}, \mathbf{d})$, transitional zone (b, e), and invaded conjunctiva (c, f). Scale bar $=25 \mu \mathrm{m}$. 


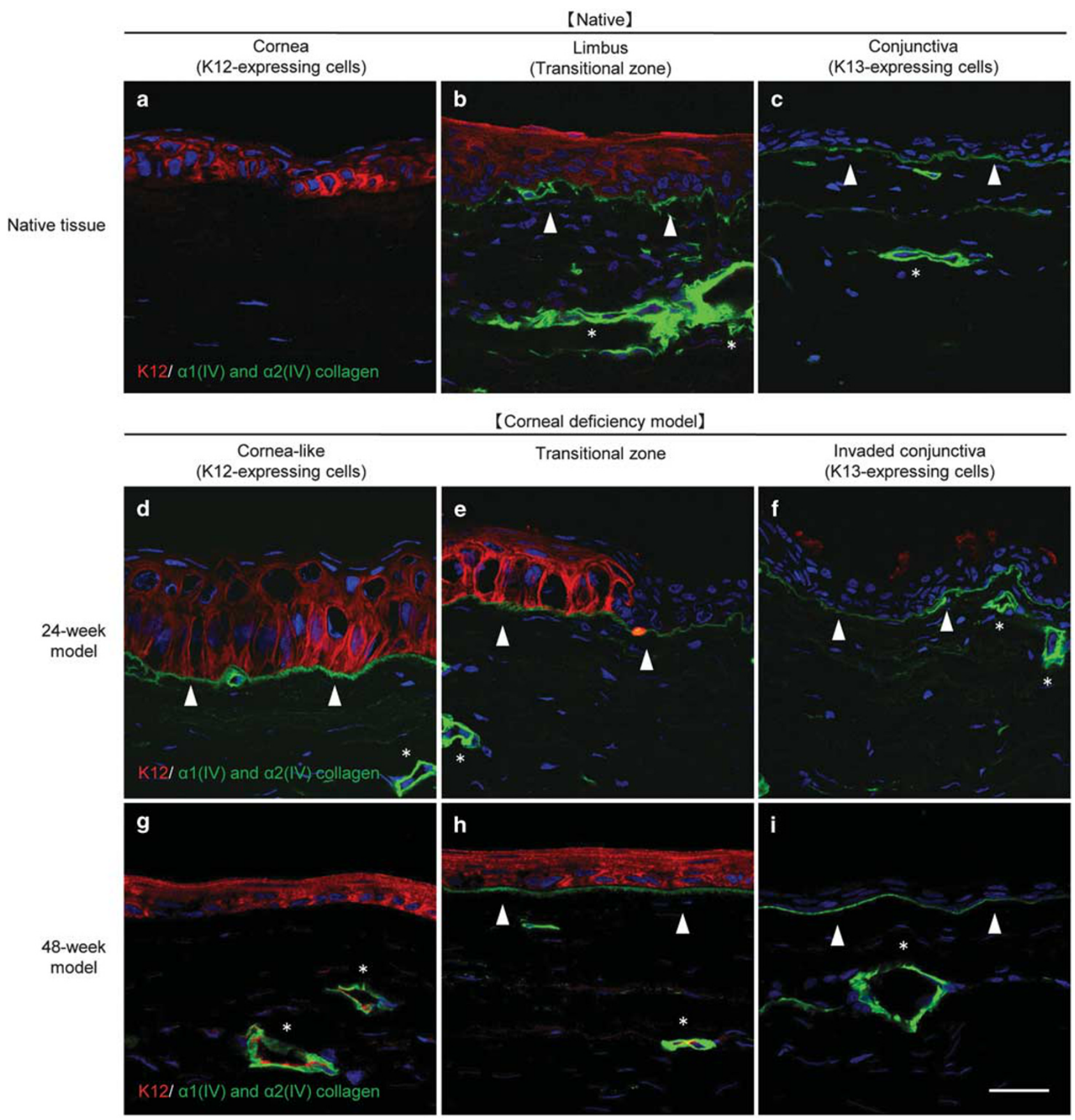

Figure 5 Changes in the distribution of $\alpha 1$ (IV) and $\alpha 2$ (IV) collagen chains beneath cornea-like cells in a corneal deficiency model. Immunohistochemistry for cytokeratin 12 (K12) (red) and $\alpha 1$ (IV) and $\alpha 2$ (IV) collagen chains (green) on native ocular surface (a-c), 24-week model surface (d-f), and 48-week model surface ( $\mathbf{g}$-i). Arrowheads indicate basement membrane containing $\alpha 1$ (IV) and $\alpha 2$ (IV) collagen chains, and asterisks indicate the vessels. Scale bar $=25 \mu \mathrm{m}$.

\section{Appearance of a Mesenchymal Cell Phenotype During the Epithelial Remodeling}

The epithelial-mesenchymal transition (EMT) is known as an induced phenomenon during the wound-healing process. $^{34}$ In this study, the corneal deficiency model specimens were stained with K14 and vimentin antibodies to investigate the induction of EMT. In the native ocular surface, K14 expression was detected only in the basal epithelial layer of the limbus, and was not observed in the central cornea and the upper epithelial cell layers of the limbus (Figures $7 \mathrm{a}-\mathrm{c}$ ). Vimentin expression was observed in keratocytes located in the corneal stroma, fibroblast cells in the connective tissue below the limbal and conjunctival epithelial cell layers, and the dendritic cells within the epi- 
thelial layer. These vimentin-expressing cells were not co-expressed with K14 on native ocular surfaces (Figures $7 \mathrm{a}-\mathrm{c}$ ). By contrast, on peripheral region of the 4 -week model surface, vimentin expression was observed not only in fibroblasts and keratocytes but also in the migrating epithelial cells. Moreover, vimentin-expressing cells were also K14-expressing

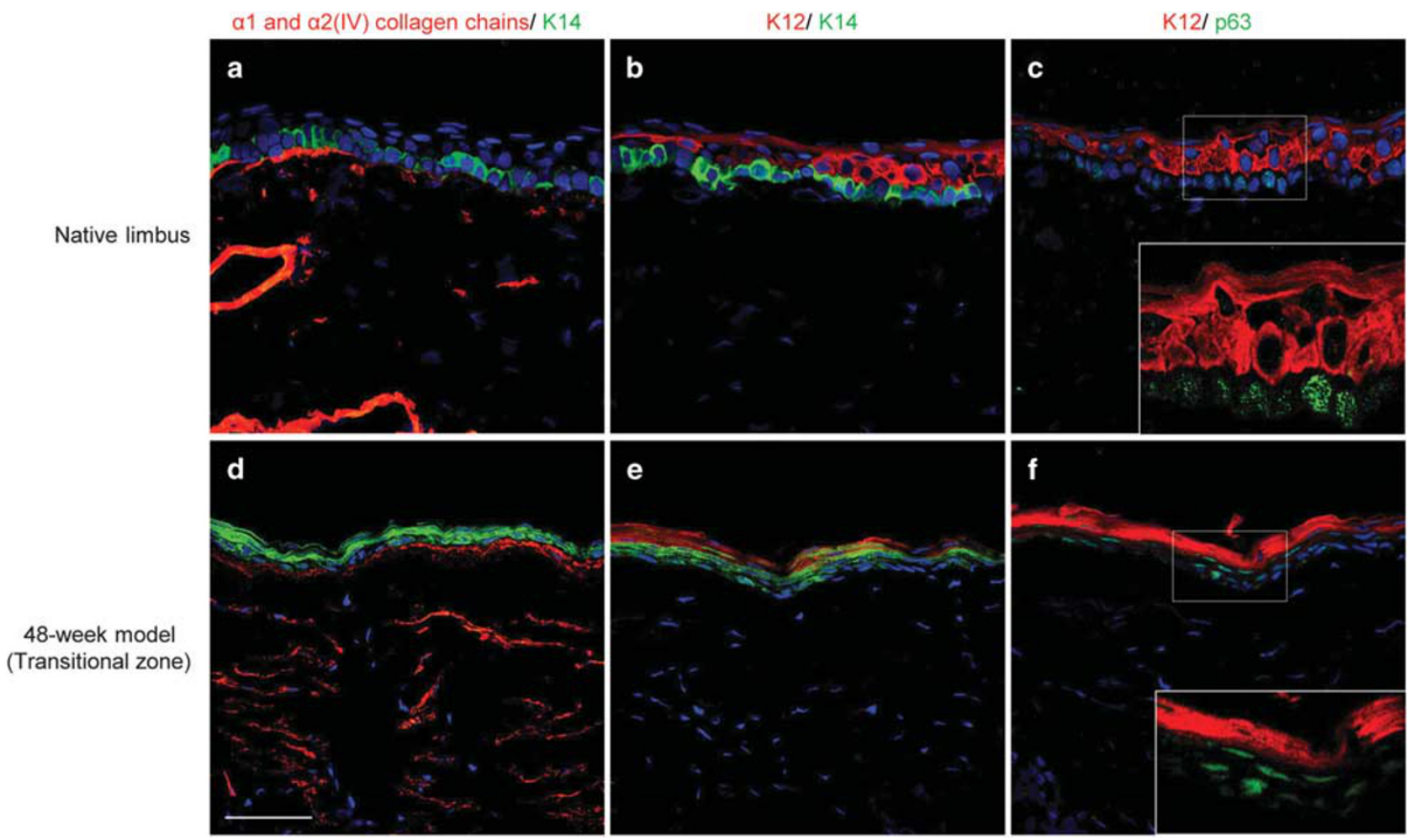

Figure 6 Reconstruction of a limbus-like structure at the transitional zone between cornea-like cells and invaded conjunctiva in the 48-week model surface. Ocular surfaces of native tissue (a-c) and 48-week model surface (d-f) are shown. Double staining of $\alpha 1$ (IV) and $\alpha 2$ (IV) collagen chains (red) and cytokeratin 14 (K14) (green; a, d), K12 (red), and K14 (green; b, e), K12 (red), and p63 (an epithelial stem/progenitor cell marker; green; c, f), respectively. High-magnification images of $\mathrm{K} 12 / \mathrm{p} 63$ staining were shown in lower right corner without DAPI staining (c, $\mathbf{f})$. Scale bar $=50 \mu \mathrm{m}$.

【Native】

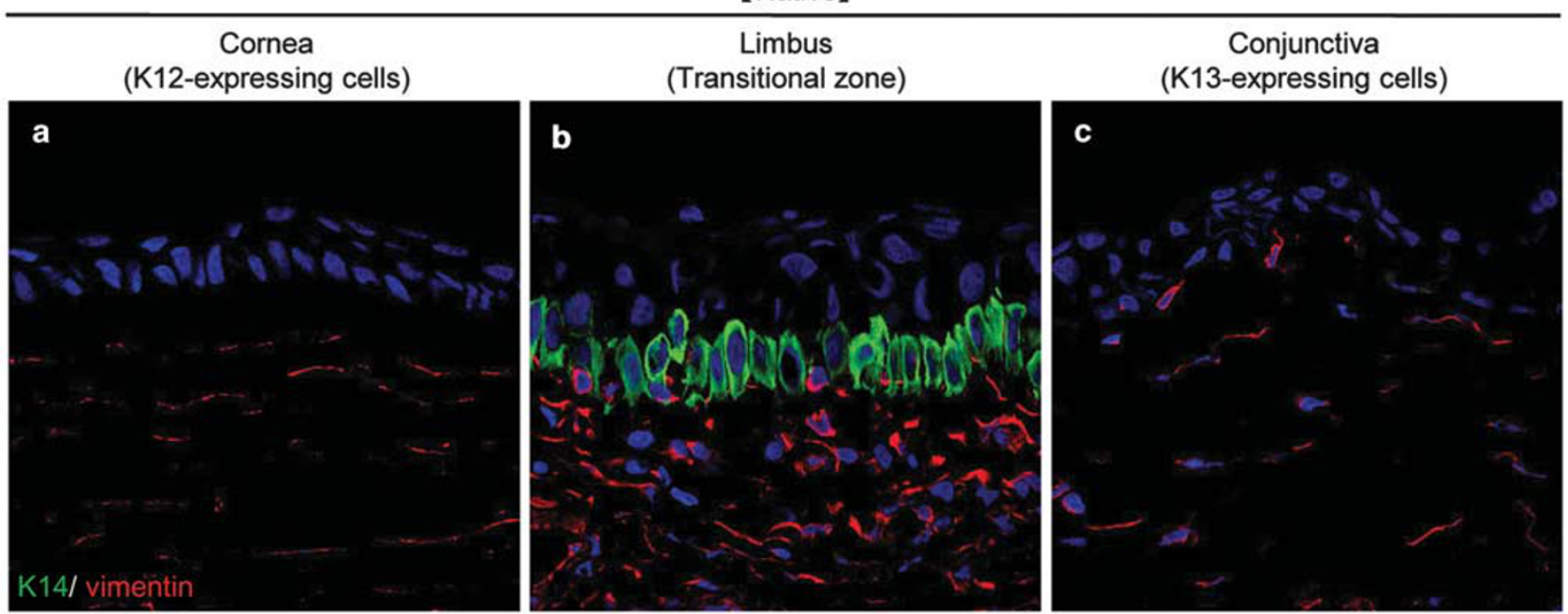

Figure 7 Analysis of cytokeratin 14 and vimentin expression on native ocular, 4-, 24-, and 48-weeks model surfaces. Double staining with cytokeratin 14 (K14), a basal epithelial cell marker, and vimentin, a mesenchymal cell marker, was performed on the native cornea, limbus, and conjunctiva, respectively $(\mathbf{a}-\mathbf{c})$. In addition, central part and peripheral region of the cornea, and conjunctival region of 4-week (d-f), 24-week (g-i), and 48-week model surfaces (j-I) are shown. White arrow heads show K14- and vimentin-co-expressing cells. Scale bar $=25 \mu \mathrm{m}$. 
【Corneal deficiency model】

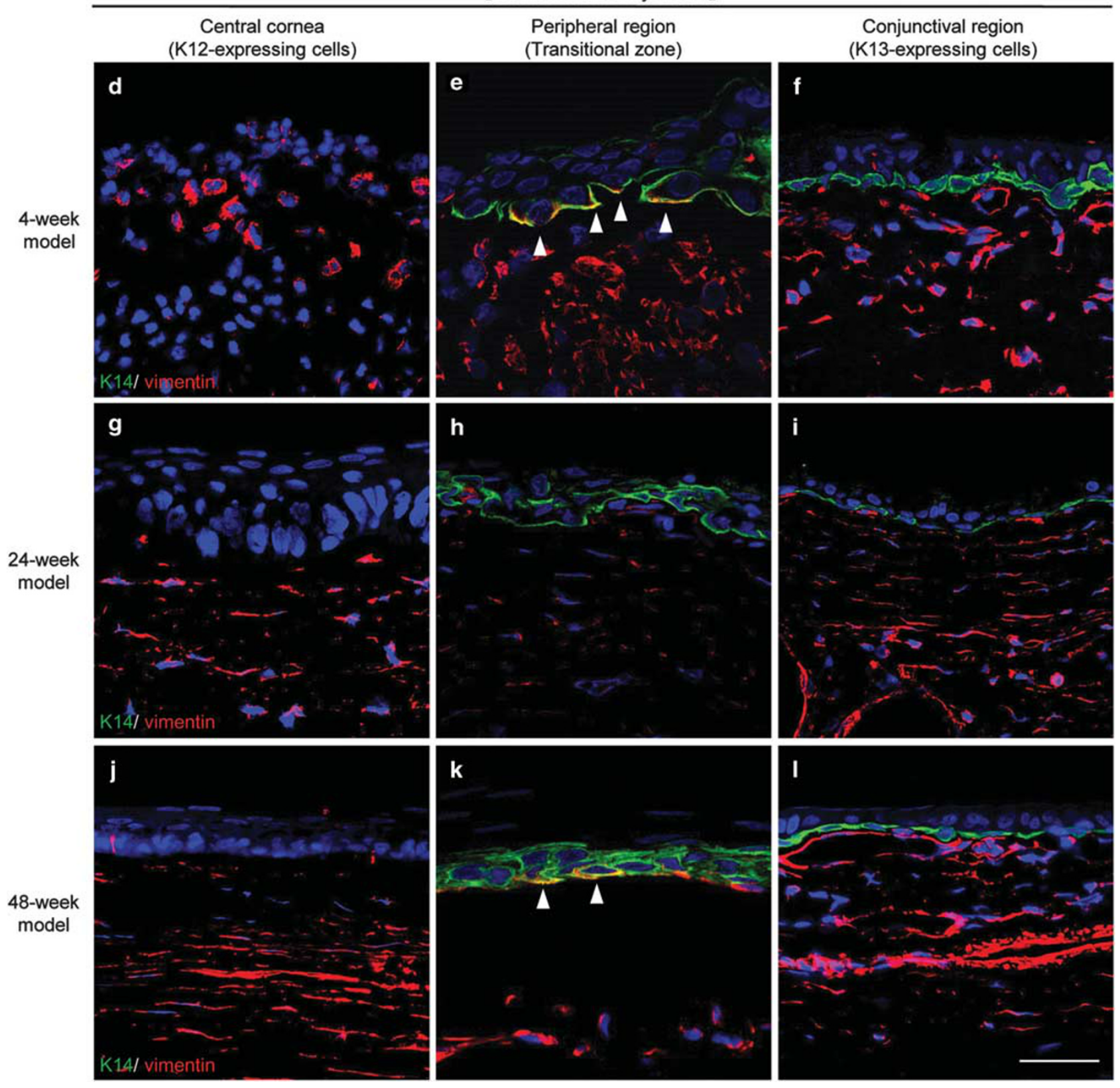

Figure 7 Continued.

cells that had migrated into the central cornea from the conjunctiva (Figure 7e, arrow head). K14 and vimentin co-expressing cells were not observed in the 4-week model surface of the conjunctival region (Figure $7 \mathrm{f}$ ), and epithelial cells had not reached the central region (Figure $7 \mathrm{~d}$ ). Furthermore, in the 48-week model surface, cells co-expressing K14 and vimentin were observed in the basal cell layer of the transitional zone between cornea-like cells and invaded conjunctiva (Figure 7k), and the co-expressing cells were not detected in cornea-like cells (Figure 7j) and invaded con- junctiva (Figure 7l). By contrast, in the 24-week model surface, K14 and vimentin co-expression was not observed in cornea-like cells (Figure $7 \mathrm{~g}$ ), the transitional zone (Figure 7h), and invaded conjunctiva (Figure 7i).

\section{DISCUSSION}

This study, using a long-term follow-up, demonstrated that K12-expressing cornea-like cells appeared on the corneal deficiency model surface. Moreover, cells expressing p63, a putative epithelial stem/progenitor cell marker, and K14, a 

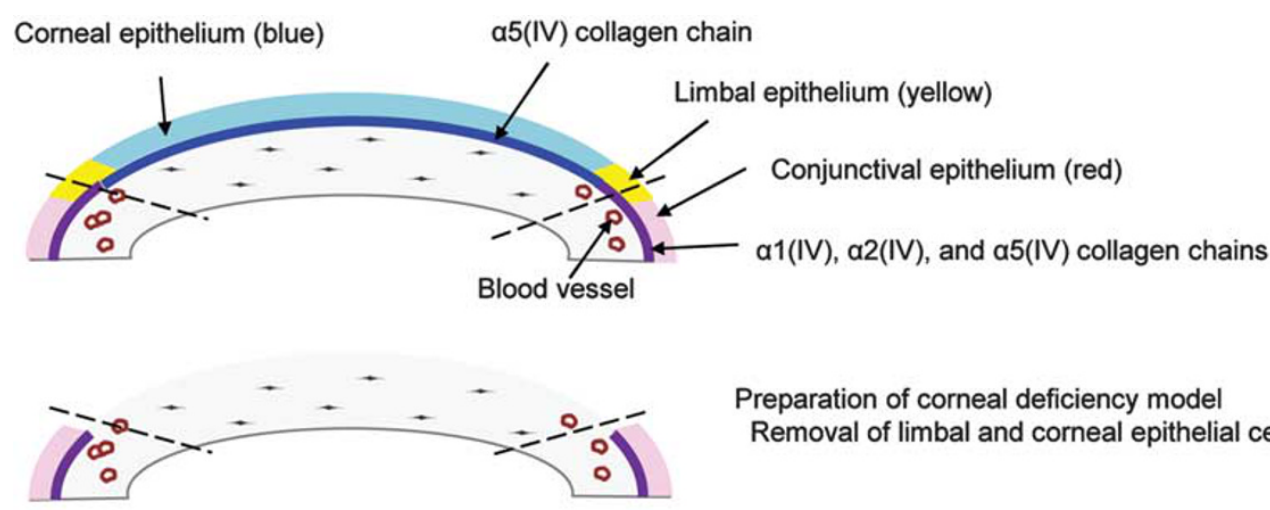

Preparation of corneal deficiency model

Removal of limbal and corneal epithelial cells and the basement membrane.
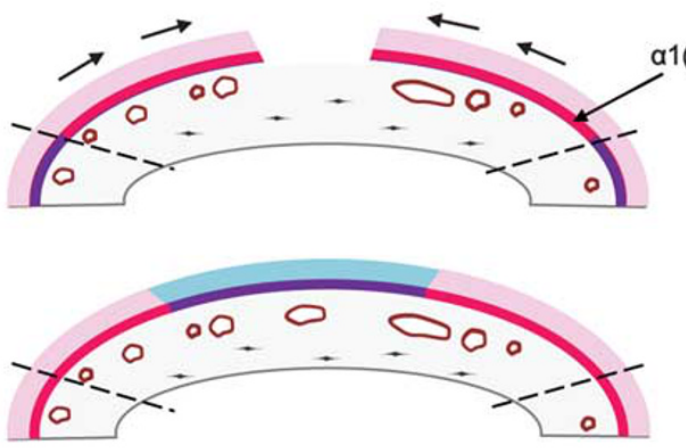

24-week model surface

The appearance of cornea-like cells (blue).
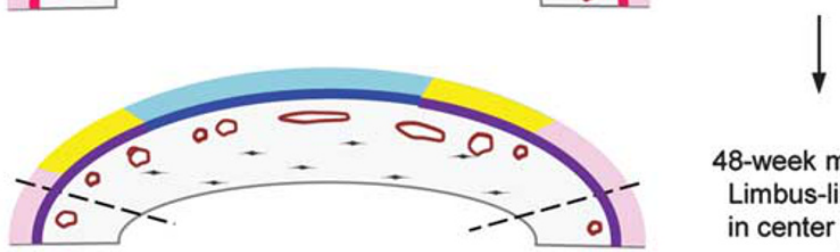

48-week model surface

Limbus-like structure (yellow) was reconstructed on the transitional zone in center part of cornea.

Figure 8 Schematic illustration of the remodeling of epithelia and basement membrane in the rabbit corneal deficiency model with long-term follow-up.

basal epithelial cell marker, were observed in the transitional zone between K12-expressing cornea-like cells and K13expressing invaded conjunctiva of the 48 -week model surface (Figure 8).

Transdifferentiation of conjunctival epithelium into cornea-like cells has been reported by observing the decrease in the number of mucin-expressing goblet cells. ${ }^{13}$ A previous study reported that cells stained positively with AE5 monoclonal antibody, which is known to recognize a major corneal 64-kD keratin (K3), are also detected in a rabbit corneal deficiency model surface. ${ }^{14}$ However, AE5 has been shown to exhibit cross-reactivity with conjunctival epithelial cells, which actually lack K3 expression. ${ }^{35}$ Recently, polyclonal antibodies against $\mathrm{K} 12$, which is specific to corneal and limbal epithelia, have been found to be useful in discriminating the corneal epithelium from the conjunctival epithelium. ${ }^{36,37}$ This study used the same goat polyclonal anti-K12 antibody ${ }^{36}$ and confirmed that this antibody demonstrated no cross-reactivity with the conjunctival epithelium and was useful to identify the corneal epithelium specifically. In this study, K12-expressing cells were found in the central part of the corneal deficiency model surface, whereas Muc5-expressing goblet cells, contained in native conjunctiva, were undetected in the cornea-like cells. Moreover, in the 24week model surface, cornea-like cells localized on the basement membrane containing $\alpha 1$ (IV) and $\alpha 2$ (IV) collagen chains, which are observed on the native limbus and conjunctiva. Although K12-expressing cells may have been generated from the remaining limbal epithelium, K13expressing conjunctival epithelium migrated and enveloped the entire corneal surfaces in the early phase of the corneal deficiency model, and some of the conjunctival epithelial cells transdifferentiated into cornea-like cells. Furthermore, cells co-expressing $\mathrm{K} 14$, an epithelial basal cell marker, and vimentin, a mesenchymal cell marker, were observed in the invaded conjunctiva in the 4-week model surface. This result suggested the induction of the EMT, which is characterized by the downregulation of the epithelial cell marker E-cadherin and the upregulation of the mesenchymal cell markers vimentin and $\mathrm{N}$-cadherin and causes morphological 
changes and enhanced migration. ${ }^{34}$ A previous study reported that EMT promotes dermal wound healing through enhancing epithelial migration in mouse keratinocytes. ${ }^{35,38}$ EMT is also reported to allow epithelial cells to acquire the stem cell phenotype. Immortalized human mammary epithelial cells transfected with Snail and Twist acquire the stem cell phenotype and sphere-formation ability through the induction of EMT. ${ }^{39}$ In addition, normal human keratinocytes transduced with $\Delta \mathrm{Np} 63$ convert their phenotype to mesenchymal cell-like and gain stem cell-like characteristics, such as the expression of Nanog and Lin28. ${ }^{40}$ Moreover, Lin28 triggers the induction of EMT and increases in mammosphere-formation ability and aldehyde dehydrogenase activity, which are considered stem/ progenitor properties. ${ }^{41,42}$ In this study, cornea-like epithelial cells had a possibility to generate from K14 and vimentin co-expressing cells, which were allowed to acquire stem cell characters through EMT. Interestingly, in the 48week model surface, cells expressing K14 and p63, a putative stem/progenitor cell marker, were detected only in the transitional zone between cornea-like cells and invaded conjunctiva, in which K14 and vimentin-co-expressing cells were also detected. Moreover, $\alpha 1$ (IV) and $\alpha 2$ (IV) collagen chains were found in the transitional zone and invaded conjunctiva and were not observed beneath cornea-like cells, which was similar to their distribution of the native ocular surface. On the basis of these results, the epithelial stem/ progenitor cells generated by EMT induction were proposed to be maintained in the transitional zone as a stem cell niche, which was similar to the native limbus. These observations indicated that conjunctival epithelial cells obtained the motility, proliferation activity, and stem cell phenotype through EMT, at an early phase of the corneal deficiency model. These cells invaded the central corneal region and entirely covered it. In the corneal stroma, cells with a stem cell phenotype were transdifferentiated into K12-expressing cornea-like cells by epithelial-mesenchymal interaction.

The molecular mechanisms of generating corneal epitheliallike cells and the accumulation of stem/progenitor cells in the transitional zone remain unclear. Recently, bone marrow cells have been reported to have the capability to contribute to the regeneration of the epidermis. ${ }^{43}$ Another investigation has reported that the fibroblast cells present in the limbal stroma have a mesenchymal stem cell phenotype ${ }^{44}$ and that these cells have the potential to differentiate into corneal epithelial cells. ${ }^{45}$ In this study, although corneal and limbal epithelia were removed during the preparation of the model, limbal fibroblast cells remained in the limbal stroma. Indeed, conjunctival epithelial cells migrated into the central corneal region with fibroblast cells. Therefore, K12-expressing cornea-like cells might have been generated from fibroblast-like cells, generated by EMT induction, on the corneal deficiency model.

The rabbit corneal deficiency model appears to be a useful tool for investigating corneal epithelial stem cells and human corneal stem cell deficiency. These observations will also eventually be useful in the development of regenerative therapies for the epithelium.

Supplementary Information accompanies the paper on the Laboratory Investigation website (http://www.laboratoryinvestigation.org)

\section{ACKNOWLEDGMENTS}

We thank Dr Norio Ueno, Dr Ryo Takagi, Mr Daisuke Murakami, and Dr Makoto Kondo (Tokyo Women's Medical University) for their useful comments and technical criticism. This study was partially supported by a Grant-in-Aid for Japan Society for the Promotion of Science (JSPS) Fellows, Creation of innovation centers for advanced interdisciplinary research areas Program in the Project for Developing Innovation Systems 'Cell Sheet Tissue Engineering Center (CSTEC)' from the Ministry of Education, Culture, Sports, Science and Technology (MEXT), Japan and the Global COE program, the Multidisciplinary Education and Research Center for Regenerative Medicine (MERCREM) from the Ministry of Education, Culture, Sports, Science and Technology (MEXT), Japan and Grant-in-Aid for Scientific Research on Innovative Areas 'Hyper Bio Assembler for 3D Cellular Innovation', Japan.

\section{DISCLOSURE/CONFLICT OF INTEREST}

Dr Teruo Okano is a founder and director of the board of Cellseed Inc. licensing technologies and patents from Tokyo Women's Medical University. Dr Teruo Okano and Dr Masayuki Yamato are shareholders of CellSeed Inc. Tokyo Women's Medical University receives research funding from Cellseed Inc.

1. Thoft RA, Friend J. Biochemical transformation of regenerating ocular surface epithelium. Invest Ophthalmol Vis Sci 1977;16:14-20.

2. Wei ZG, Wu RL, Lavker RM, et al. In vitro growth and differentiation of rabbit bulbar, fornix, and palpebral conjunctival epithelia. Implications on conjunctival epithelial transdifferentiation and stem cells. Invest Ophthalmol Vis Sci 1993;34:1814-1828.

3. Wei ZG, Sun TT, Lavker RM. Rabbit conjunctival and corneal epithelial cells belong to two separate lineages. Invest Ophthalmol Vis Sci 1996:37:523-533.

4. Thoft RA, Friend J. The $X, Y, Z$ hypothesis of corneal epithelial maintenance. Invest Ophthalmol Vis Sci 1983;24:1442-1443.

5. Buck RC. Measurement of centripetal migration of normal corneal epithelial cells in the mouse. Invest Ophthalmol Vis Sci 1985;26: 1296-1299.

6. Kinoshita S, Friend J, Thoft RA. Sex chromatin of donor corneal epithelium in rabbits. Invest Ophthalmol Vis Sci 1981;21:434-441.

7. Kenyon KR, Tseng SC. Limbal autograft transplantation for ocular surface disorders. Ophthalmology 1989;96:709-722.

8. Tsubota K, Satake $Y$, Kaido M, et al. Treatment of severe ocular-surface disorders with corneal epithelial stem-cell transplantation. N Engl J Med 1999;340:1697-1703.

9. Tsai RJ, Sun TT, Tseng SC. Comparison of limbal and conjunctival autograft transplantation in corneal surface reconstruction in rabbits. Ophthalmology 1990;97:446-455.

10. Shapiro MS, Friend J, Thoft RA. Corneal re-epithelialization from the conjunctiva. Invest Ophthalmol Vis Sci 1981;21:135-142.

11. Dua HS. The conjunctiva in corneal epithelial wound healing. $\mathrm{Br} J$ Ophthalmol 1998;82:1407-1411.

12. Ti SE, Anderson D, Touhami A, et al. Factors affecting outcome following transplantation of ex vivo expanded limbal epithelium on amniotic membrane for total limbal deficiency in rabbits. Invest Ophthalmol Vis Sci 2002;43:2584-2592.

13. Kinoshita S, Friend J, Thoft RA. Biphasic cell proliferation in transdifferentiation of conjunctival to corneal epithelium in rabbits. Invest Ophthalmol Vis Sci 1983;24:1008-1014.

14. Kruse FE, Chen JJ, Tsai RJ, et al. Conjunctival transdifferentiation is due to the incomplete removal of limbal basal epithelium. Invest Ophthalmol Vis Sci 1990;31:1903-1913. 
15. Li W, Hayashida Y, Chen YT, et al. Niche regulation of corneal epithelial stem cells at the limbus. Cell Res 2007;17:26-36.

16. Li DQ, Tseng SC. Three patterns of cytokine expression potentially involved in epithelial-fibroblast interactions of human ocular surface. J Cell Physiol 1995;163:61-79.

17. Ihanamäki T, Pelliniemi LJ, Vuorio E. Collagens and collagen-related matrix components in the human and mouse eye. Prog Retin Eye Res 2004;23:403-434.

18. Saharinen J, Hyytiäinen M, Taipale J, et al. Latent transforming growth factor-beta binding proteins (LTBPs)-structural extracellular matrix proteins for targeting TGF-beta action. Cytokine Growth Factor Rev 1999:10:99-117.

19. Schlötzer-Schrehardt U, Dietrich T, Saito K, et al. Characterization of extracellular matrix components in the limbal epithelial stem cell compartment. Exp Eye Res 2007;85:845-860.

20. Sugiyama $H$, Yamato $M$, Nishida $K$, et al. Evidence of the survival of ectopically transplanted oral mucosal epithelial stem cells after repeated wounding of cornea. Mol Ther 2014;22:1544-1555.

21. Ninomiya $Y$, Kagawa $M$, lyama $K$, et al. Differential expression of two basement membrane collagen genes, COL4A6 and COL4A5, demonstrated by immunofluorescence staining using peptidespecific monoclonal antibodies. J Cell Biol 1995;130:1219-1229.

22. Satake $Y$, Dogru M, Yamane GY, et al. Barrier function and cytologic features of the ocular surface epithelium after autologous cultivated oral mucosal epithelial transplantation. Arch Ophthalmol 2008;126:23-28.

23. Meek KM, Leonard DW. Ultrastructure of the corneal stroma: a comparative study. Biophys J 1993:64:273-280.

24. Marshall GE, Konstas AG, Lee WR. Collagens in ocular tissues. $\mathrm{Br} J$ Ophthalmol 1993;77:515-524.

25. Espana EM, Di Pascuale MA, He $\mathrm{H}$, et al. Characterization of corneal pannus removed from patients with total limbal stem cell deficiency. Invest Ophthalmol Vis Sci 2004;45:2961-2966.

26. Tanioka H, Kawasaki S, Yamasaki K, et al. Establishment of a cultivated human conjunctival epithelium as an alternative tissue source for autologous corneal epithelial transplantation. Invest Ophthalmol Vis Sci 2006:47:3820-3827.

27. Majo F, Rochat A, Nicolas M, et al. Oligopotent stem cells are distributed throughout the mammalian ocular surface. Nature 2008;456: 250-254.

28. Inatomi T, Spurr-Michaud S, Tisdale AS, et al. Expression of secretory mucin genes by human conjunctival epithelia. Invest Ophthalmol Vis Sci 1996:37:1684-1692.

29. Puangsricharern V, Tseng SC. Cytologic evidence of corneal diseases with limbal stem cell deficiency. Ophthalmology 1995;102:1476-1485.

30. Espana EM, Grueterich $M$, Romano AC, et al. Idiopathic limbal stem cell deficiency. Ophthalmology 2002;109:2004-2010.
31. Thorner PS, Zheng K, Kalluri R, et al. Coordinate gene expression of the alpha3, alpha4, and alpha5 chains of collagen type IV. Evidence from a canine model of X-linked nephritis with a COL4A5 gene mutation. J Biol Chem 1996;271:13821-13828.

32. Guerriero E, Chen J, Sado $Y$, et al. Loss of alpha3(IV) collagen expression associated with corneal keratocyte activation. Invest Ophthalmol Vis Sci 2007;48:627-635.

33. Schlötzer-Schrehardt U, Kruse FE. Identification and characterization of limbal stem cells. Exp Eye Res 2005;81:247-264.

34. Aomatsu K, Arao T, Abe K, et al. Slug is upregulated during wound healing and regulates cellular phenotypes in corneal epithelial cells. Invest Ophthalmol Vis Sci 2012;53:751-756.

35. Barbaro V, Ferrari S, Fasolo A, et al. Evaluation of ocular surface disorders: a new diagnostic tool based on impression cytology and confocal laser scanning microscopy. Br J Ophthalmol 2010;94:926-932.

36. Boehlke CS, Yuan C, Kao WW, et al. Cytokeratin 12 in human ocula surface epithelia is the antigen reactive with a commercial anti-Galpha q antibody. Mol Vis 2004;10:867-873.

37. Kawasaki S, Tanioka H, Yamasaki K, et al. Clusters of corneal epithelial cells reside ectopically in human conjunctival epithelium. Invest Ophthalmol Vis Sci 2006;47:1359-1367.

38. Terao M, Ishikawa A, Nakahara S, et al. Enhanced epithelialmesenchymal transition-like Phenotype in N-Acetylglucosaminyltransferase $\mathrm{V}$ transgenic mouse skin promotes wound healing. J Biol Chem 2011:286:28303-28311.

39. Sendurai AM, Wenjun G, Mai-Jing L, et al. The epithelial-mesenchymal transition generates cells with properties of stem cells. Cell 2008;16: 704-715.

40. Oh JE1 Kim RH, Shin KH, et al. DeltaNp63 $\alpha$ protein triggers epithelialmesenchymal transition and confers stem cell properties in normal human keratinocytes. J Biol Chem 2011:286:38757-38767.

41. Liu Y, Li H, Feng J, et al. Lin28 induces epithelial-to-mesenchymal transition and stemness via downregulation of let-7a in breast cancer cells. PLoS ONE 2013;8:e83083.

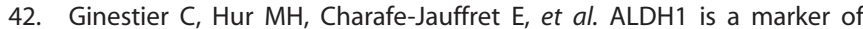
normal and malignant human mammary stem cells and a predictor of poor clinical outcome. Cell Stem Cell 2007;1:555-567.

43. Tamai K, Yamazaki T, Chino T, et al. PDGFR $\alpha$-positive cells in bone marrow are mobilized by high mobility group box 1 (HMGB1) to regenerate injured epithelia. Proc Natl Acad Sci USA 2011;16: 6609-6614.

44. Polisetty N, Fatima A, Madhira SL, et al. Mesenchymal cells from limbal stroma of human eye. Mol Vis 2008;14:431-442.

45. Katikireddy KR, Dana R, Jurkunas UV. Differentiation potential of limbal fibroblasts and bone marrow mesenchymal stem cells to corneal epithelial cells. Stem Cells 2013;32:717-729. 